\title{
Der Bürgerdialog Zukunftstechnologien des BMBF
}

\author{
Cornelia Quennet-Thielen
}

\begin{abstract}
Kernaussagen
Der rasche gesellschaftliche Wandel macht zusätzliche Interaktionsformen zwischen Bürgern, Politik und Verwaltung erforderlich. Die Bundesregierung hat in den vergangenen Jahren eine Reihe von Bürgerdialogen initiiert, wie die Bürgerdialoge über Zukunftstechnologien des BMBF. Diese Bürgerdialoge bieten besondere Chancen, im Sinne mündiger Bürger und einer reflektierenden Politik Verantwortung zu übernehmen: Einblick zu gewinnen, Position zu beziehen, Konflikte konstruktiv zu diskutieren und gemeinsam an der Gestaltung der Zukunft mitzuwirken. Die Ergebnisse fließen nun in den politischen Entscheidungsprozess ein. Sie wurden in Bundesministerien, den Bundestag und die Bundesländer, in die wissenschaftlichen Beratungsgremien zur Forschungspolitik, in Organisationen und Unternehmen sowie in die Öffentlichkeit kommuniziert. Hierbei geht es nicht allein darum, eine berechtigte Erwartung der Bürger zu erfüllen, sondern auch um eine qualitative Verbesserung politischer Entscheidungen, indem Know-how und Beurteilungsfähigkeiten der Bürger genutzt werden.
\end{abstract}

„Es ist eine Irrlehre, dass es Fragen gibt, die für den normalen Bürger zu groß und zu kompliziert seien [...]. Politik ist zugänglich, beeinflussbar für jeden. Das ist der zentrale Punkt der Demokratie. “

Olof Palme, schwedischer Ministerpräsident

\section{Bürgerdialoge}

Die repräsentative Demokratie steht vor großen Herausforderungen. In Zeiten raschen Wandels, globaler Vernetzung und komplexer Zusammenhänge ist es schwieriger geworden, tragfähige Lösungen auf der Grundlage gesicherten Wissens zu entwickeln und dafür gesellschaftliche Zustimmung zu finden. Das Bedürfnis vieler Bürgerinnen und Bürger nach Partizipation jenseits des Rechts, zu wählen und gewählt zu werden, wächst. Während wissenschaftliche Politikberatung, Ressortforschung und Beratungsgremien bewährte Instrumente sind, um die inhaltlichen Basis für politische Entscheidungen $\mathrm{zu}$ verbreitern und unterschiedliche Handlungsoptionen gegeneinander abzuwägen, haben Politik und Verwaltung ungleich weniger Erfahrung damit, direkt mit den Bürgerinnen und Bürgern in den Dialog über Zukunftsfragen zu treten. Aber auch hier geschieht inzwischen viel.

Die Bundesregierung hat in den vergangenen Jahren eine Reihe von Dialogformaten initiiert: Vom Zukunftsdialog der
Bundeskanzlerin über den Nachhaltigkeitsdialog der Bundesregierung, den Pflegedialog des Bundesministeriums für Gesundheit bis zum dialog-internet, in dessen Mittelpunkt die Kinder- und Jugendnetzpolitik des Bundesministeriums für Familie, Senioren, Frauen und Jugend steht.

Das Bundesministerium für Bildung und Forschung ist einer der Vorreiter eines breit angelegten Bürgerdialogs zu zentralen gesellschaftlichen Fragen. Mit den Zukunftstechnologien greifen wir in unseren Bürgerdialogen Themen auf, bei denen großer Informations- und Diskussionsbedarf besteht. Dabei zielen wir nicht nur auf breite Beteiligungsmöglichkeiten, sondern auch auf konkrete Empfehlungen. Demokratie lebt von urteilsfähigen Bürgerinnen und Bürgern, die sich einbringen. „Ihr werdet Euch noch wünschen, wir wären politikverdrossen“ - das bekannte Zitat eine Bloggers muss uns Ansporn sein. In Bürgerdialogen können konkrete Erfahrungen und Bedürfnisse vor Ort früher und deutlicher artikuliert, sensible Punkte frühzeitiger eingebracht und die Basis für Entscheidungen verbreitert werden. So kann die oft beklagte Kluft zwischen politisch Verantwortlichen, Engagierten und Betroffenen verringert werden, allerdings nur, wenn von vornherein klar ist, worum es geht und was das Ziel ist. Wie erreichen wir, dass alle ähnlich gut informiert sind? Um welche Frage geht es genau? Wer kann sich wie beteiligen? Wie vermeiden wir, dass der Dialog als „Show“ oder „Alibiveranstaltung“ wahrgenommen wird? Wie gestalten wir einen konstruktiven kommunikativen Prozess?

Bürgerbeteiligung ersetzt nicht die politische Entscheidung. Demokratisch legitimierte Politikerinnen und Politiker müssen verantwortlich und handlungsfähig bleiben. Weder können sie Verantwortung abwälzen, noch können demokratisch nicht oder nur schwach legitimierten Gruppen diese übernehmen. Entscheidungen müssen von denen getroffen werden, die mit dem treffenden deutschen Wort als „Volksvertreter" bezeichnet werden. Bürger zu beteiligen bedeutet allerdings sehr wohl, das Fundament für politische Entscheidungen zu verbreitern und die Resonanz zu verstärken.

\section{Bürgerdialog über Zukunftstechnologien}

Zukunftstechnologien helfen uns, zentrale Herausforderungen der Gegenwart zu lösen - von einer modernen und menschlichen Medizin über eine sichere, günstige und umweltschonende Energieversorgung bis hin zu innovativer und leistungsfähiger Mobilität. Gleichzeitig können sie weitreichende Konsequenzen für jeden Einzelnen haben. Häufig sind sie mit gesellschaftlichen Weichenstellungen verbunden. Nutzen und mögliche Risiken sind nicht immer leicht abzusehen. Faszination, Angst und Unsicherheit liegen nahe beieinander. Oft geht es dabei weniger um das Für und Wider neuer Technologien, 
sondern um das Wie ihrer Anwendung, um das Verhältnis von Nutzen und möglichen Nachteilen oder um unbeabsichtigte Folgen. Die Diskussion über diese Fragen erfordert vertiefte, technische Informationen und wirft oft Grundfragen auf: Wie beherrschbar ist eine Technologie? Welchen Fortschrittsbegriff haben wir? In welcher Gesellschaft wollen wir zukünftig leben?

Ein Bürgerdialog über Zukunftstechnologien bietet daher besondere Chancen, im Sinne mündiger Bürger und einer reflektierenden Politik Verantwortung zu übernehmen: Einblick zu gewinnen, Position zu beziehen, Konflikte konstruktiv zu diskutieren und gemeinsam an der Gestaltung der Zukunft unseres Landes mitzuwirken. Deshalb haben die Regierungsparteien im Koalitionsvertrag beschlossen, einen umfassenden Dialog über Zukunftstechnologien zu führen, in dem Bürgerinnen und Bürger sich über aktuelle Forschung auf zukunftsweisenden Gebieten informieren und sich im offenen Austausch mit Experten eine fundierte Meinung dazu bilden können. Sie sollen Entscheidungsträgern aus Politik, Wissenschaft und Wirtschaft begegnen und mit ihnen über ihre Erwartungen und Besorgnisse diskutieren können. Gleichzeitig sollen sie konkrete Empfehlungen an Wissenschaft und Politik formulieren und so den Umgang mit den diskutierten Zukunftstechnologien mit prägen können.

Damit ein solcher Dialog gelingt, müssen Abläufe und Regeln so gestaltet sein, dass ein echter Informations- und Meinungsaustausch möglich wird. Offenheit und Transparenz sind erforderlich, um einer breiten Vielfalt von Meinungen und Perspektiven Gehör zu verschaffen. Konflikte müssen konstruktiv gelöst und Diskussionen sorgfältig und neutral moderiert werden. Dabei stand dem BMBF bei der Konzeption und der Durchführung der Dialoge mit der IFOK GmbH ein Partner zur Seite, der bereits umfassende Erfahrungen in Bürgerbeteiligungsprozessen, insbesondere bei Infrastrukturgroßprojekten gesammelt hat [vgl. Beitrag in diesem Heft].

Wissenschaftliche Experten werden in den gesamten Dialog-Prozess eingebunden, damit die Teilnehmerinnen und Teilnehmer für ihre Diskussion genaue und aktuelle Informationen erhalten. Eine kontinuierliche Bewertung des DialogProzesses sorgt dafür, dass er an die Bedürfnisse der Teilnehmerinnen und Teilnehmer und die Erfordernisse des Themas angepasst werden kann. Dies gelingt durch ein gestaffeltes Verfahren. Als Diskussionsgrundlage werden in einer ersten Phase Impulspapiere verfasst. Sie werden von Fachleuten erarbeitet und anschließend im Rahmen von Fokusgruppen mit zufällig ausgewählten Bürgern reflektiert und ergänzt. Zusätzlich finden offene Bürgerwerkstätten statt. Diese Abendveranstaltungen sind für alle interessierten Bürger offen und bieten ein breites lokales Stimmungsbild, das dann in den weiteren Prozess einfließt. Kernelement des Dialogs sind die regionalen „Bürgerkonferenzen“ als zentrale Foren des Wissensaustausches und der Diskussion. Jeweils 100 zufällig ausgewählte Bürgerinnen und Bürger diskutieren die Chancen und Herausforderungen einer Zukunftstechnologie. Sie entwickeln erste Lösungs- und Handlungsansätze, die in regionalen
Zwischenberichten gesammelt werden. Parallel findet ein Online-Dialog statt, an dem sich alle Bürgerinnen und Bürger beteiligen können. Die hier abgegebenen Bewertungen fließen ebenfalls in die Berichte ein.

Alle Materialien werden auf einem abschließenden nationalen Bürgergipfel in Berlin von einhundert Bürgerinnen und Bürgern diskutiert, die zuvor an einer Bürgerkonferenz teilgenommen haben. Sie erarbeiten in zweitägigen Beratungen den abschließenden Bürgerreport. Dieser fasst zusammen, wo die Bürgerinnen und Bürger Chancen und Herausforderungen sowie relevante Handlungsfelder und Fragestellungen zu Zukunftstechnologien sehen. Er wird am Ende des Prozesses an Entscheidungsträger aus Politik, Wirtschaft, Wissenschaft und Gesellschaft übergeben und fließt in vielfältige Erörterungen und Entscheidungsprozesse ein.

Den gesamten Prozess begleitet ein Beraterkreis. Hier finden sich Experten aus Politik, Verwaltung, Wissenschaft, Wirtschaft und Zivilgesellschaft zusammen, die zusätzliches Wissen und ein breites Meinungsspektrum beisteuern. Gleichzeitig fungieren die Mitglieder des Beraterkreises als Botschafter des Bürgerdialogs in ihre Institutionen und Communities.

\section{Konkrete Inhalte und Erfahrungen}

Bisher haben zwei Bürgerdialoge des BMBF stattgefunden: „Energietechnologien für die Zukunft“ und „Hightech-Medizin“. Insgesamt fanden 14 Bürgerkonferenzen, 22 Bürgerwerkstätten und zwei Bürgergipfel mit insgesamt über 2.000 Bürgerinnen und Bürgern statt. Darüber hinaus beteiligten sich rund 10.000 Menschen an den begleitenden Online-Dialogen. Die einzelnen Phasen der Veranstaltungen wurden durchweg positiv wahrgenommen. Die meisten Teilnehmer schätzten die Bedeutung von Bürgerbeteiligungsverfahren hoch ein und sahen hierin einen effektiven Weg, die Qualität politischer Entscheidungen zu verbessern. Viele betonten die Chance, sich vor Ort im direkten Austausch mit Experten vertieft informieren zu können. So erklärten fast alle Teilnehmer des Dialogs zu Energietechnologien, dieser habe ihnen die Möglichkeit eröffnet, einen klaren Standpunkt zur Zukunft der Energie zu beziehen und ihre eigene Meinung einzubringen. Sie wollten das Thema in Zukunft vertieft politisch verfolgen und an weiteren Veranstaltungen dieser Art teilnehmen.

Dabei war das Verständnis der Rollenverteilung zwischen Bürgern und Politik je nach Thema durchaus unterschiedlich: Während der Politik im Bereich der Energietechnologien eine aktive Rolle zugewiesen wurde, um innovative Wege der Energiegewinnung auf den Weg zu bringen, stellten die Diskussionen zur Hightech-Medizin stärker den und die Einzelne in den Mittelpunkt. Der Politik wurde mehr die Rolle zugewiesen, gute Rahmenbedingungen zu schaffen als aktive Förderung bestimmter Technologien zu betreiben.

Eine Herausforderung bestand darin, Teilnehmerinnen und Teilnehmer aus allen Teilen und Altersgruppen der Gesellschaft für den Bürgerdialog zu gewinnen, was insbesondere im 
Fall jüngerer und nicht-akademisch gebildeter Personen kein leichtes Unterfangen war. Die andere Herausforderung bestand darin, den Online-Dialog, an dem sich zahlreiche Personen beteiligten, $\mathrm{zu}$ moderieren, die vielen Anfragen $\mathrm{zu}$ beantworten und die Eintragungen zu kategorisieren, damit sie im Bürgerreport berücksichtigt werden konnten.

Im Bürgerdialog Energietechnologien legten die meisten Teilnehmer einen Schwerpunkt auf die erneuerbaren Energien. Eine große Mehrheit sprach sich für die Weiterentwicklung von Solar- und Windenergie aus. Überraschend war, wie sehr immer wieder eine kommunale respektive „rekommunalisierte“ Energieversorgung gefordert wurde. Im Bürgerdialog zur Hightech-Medizin standen Fragen wie gesund sein und bleiben, gut beraten und behandelt werden sowie Begleitung beim Sterben im Mittelpunkt. Gefordert wurde mehr Raum für menschliche Betreuung und persönlichen Kontakt sowie eine Verbesserung der Arbeitsbedingungen in der Intensivund Palliativmedizin. Unerwartet deutlich sprachen sich die Bürger für die in der öffentlichen Wahrnehmung und der politischen Debatte durchaus umstrittene elektronische Gesundheitskarte aus, für den Einsatz der Telemedizin gerade in ländlichen Räumen sowie für eine stärkere Nutzung von Patientenverfügung und Vorsorgevollmacht. Patientenaufklärung, Gesundheitsbildung und unabhängig finanzierte Gesundheitsforschung sollen ausgebaut werden. Forschung im Bereich Hightech-Medizin wurde überwiegend positiv beurteilt, vielfach war die Zugangsgerechtigkeit Thema und der Aufbau transparenter Entscheidungsstrukturen. (Weitere Informationen unter: www.buergerdialog-bmbf.de).

\section{Fazit}

Die Ergebnisse der beiden ersten Bürgerdialoge Zukunftstechnologien fließen nun in den politischen Entscheidungsprozess ein: sie wurden breit kommuniziert, in Bundesministerien, den Bundestag und die Bundesländer, in die wissenschaftlichen Beratungsgremien zur Forschungspolitik, in Organisationen und Unternehmen sowie in die Öffentlichkeit. Hierbei geht es nicht allein darum, eine berechtigte Erwartung der Bürger zu erfüllen, sondern auch um eine qualitative Verbesserung politischer Entscheidungen, indem Know-how und Beurteilungsfähigkeiten der Bürger genutzt werden. „Aus Betroffenen Beteiligte machen “ - so lässt sich dieser Aspekt des Bürgerdialogs umschreiben. Nach Abschluss des Dialogs wird der Kontakt mit den beteiligten Bürgern online über einen Newsletter sowie über Einladungen zu Beraterkreisen und Fachveranstaltungen weiter gepflegt. Innerhalb des BMBF werden die Ergebnisse einbezogen in den Arbeitsprozess: von der Entwicklung konkreter Förderaktivitäten für Forschung und Entwicklung bis zur Ausrichtung der Wissenschaftskommunikation.

Bürgerbeteiligung ist eine Ressource, die Gesellschaft und Politik hohen Nutzen bringt - wenn sie mit Sorgfalt, Umsicht und Respekt erfolgt. Die Bürgerdialoge des BMBF haben gezeigt, mit welche hohem Engagement, vertiefter Kenntnis und scharfem Blick Bürgerinnen und Bürger über Technolo- gien von morgen beraten und urteilen. Die Dialogprozesse sind in konkrete Handlungsempfehlungen gemündet, die in Wirtschaft, Wissenschaft und Politik mit hohem Interesse aufgenommen wurden. Die Beteiligten sind mehrheitlich hoch motiviert, auch in Zukunft bei wesentlichen Fragen unseres Gemeinwesens mitzuwirken. Eine Herausforderung für die Zukunft bleibt, noch stärker Personen aus allen Teilen der Gesellschaft in den Dialog einzubeziehen und die längerfristige Wirksamkeit der Empfehlungen wie auch die Nachhaltigkeit für die Teilnehmer sicherzustellen.

Das BMBF wird die Reihe der Bürgerdialoge 2012 fortsetzen - als Ausdruck für mehr Partizipation und für eine zugängliche und gestaltbare Politik und als Beitrag zur gesellschaftlichen Debatte über Zukunftsthemen wie zur gelebten Demokratie.

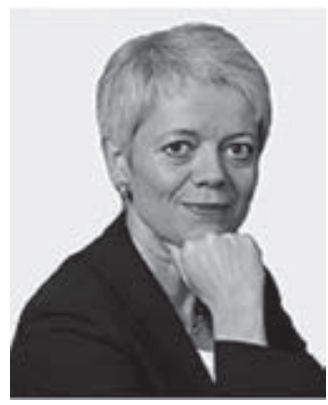

Cornelia Quennet-Thielen ist seit 2008 Staatssekretärin und Amtschefin des Bundesministeriums für Bildung und Forschung. Sie ist u.a. Mitglied in der Verwaltungskommission des Wissenschaftsrates, Mitglied des Senats der LeibnizGemeinschaft (WGL) sowie Kuratoriumsvorsitzende der Deutsch-Polnischen Wissenschaftsstiftung (DPWS). E-Mail: bmbf@bmbf.bund.de 